\title{
Bosonic String and String Field
}

\author{
Theory: a solution using
}

\section{Ultradistributions of Exponential}

\author{
Type *
}

\author{
C.G.Bollini and M.C.Rocca \\ Departamento de Física, Fac. de Ciencias Exactas, \\ Universidad Nacional de La Plata. \\ C.C. 67 (1900) La Plata. Argentina.
}

Ferbruary 1, 2007

\footnotetext{
Abstract

${ }^{*}$ This work was partially supported by Consejo Nacional de Investigaciones Cientificas and Comisión de Investigaciones Científicas de la Pcia. de Buenos Aires; Argentina.
} 
In this paper we show that Ultradistributions of Exponential Type (UET) are appropriate for the description in a consistent way string and string field theories. A new Lagrangian for the closed string is obtained and shown to be equivalent to Nambu-Goto's Lagrangian. We also show that the string field is a linear superposition of UET of compact support CUET). We evaluate the propagator for the string field, and calculate the convolution of two of them.

PACS: 03.65.-w, 03.65.Bz, 03.65.Ca, 03.65.Db. 


\section{Introduction}

In a serie of papers [1, 2, 3, 4, 5] we have shown that Ultradistribution theory of Sebastiao e Silva [6, 7, 8] permits a significant advance in the treatment

of quantum field theory. In particular, with the use of the convolution of Ultradistributions we have shown that it is possible to define a general product of distributions ( a product in a ring with divisors of zero) taht sheds new ligth on the question of the divergences in Quantum Field Theory. Furthermore, Ultradistributions of Exponential Type (UET) are adequates to describe Gamow States and exponentially increasing fields in Quantun Field Theory [9, 10, 11].

Ultradistributions also have the advantage of being representable by means of analytic functions. So that, in general, they are easier to work with and, as we shall see, have interesting properties. One of those properties is that Schwartz's tempered distributions are canonical and continuously injected into Ultradistributions of Exponential Type and as a consequence the Rigged Hilbert Space with tempered distributions is canonical and continuously included in the Rigged Hilbert Space with Ultradistributions of Exponential Type.

Another interesting property is that the space of UET is reflexive under 
the operation of Fourier transform (in a similar way of tempered distributions of Schwartz)

In this paper we show that Ultradistributions of Exponential type provides an adecuate framework for a consistent treatment of string and string field theories. In particular, a general state of the closed bosonic bradyonic string is represented by UET of compact support, and as a consequence the string field of a bradyonic bosonic string is a linear combination of UET of compact support (CUET).

This paper is organized as follows: in sections 2 and 3 we define the Ultradistributions of Exponential Type and their Fourier transform. They are part of a Guelfand's Triplet ( or Rigged Hilbert Space [12] ) together with their respective duals and a "middle term" Hilbert space. In section 4 we treate the question of the equivalence of Nanbu-Goto Lagrangian with a new Lagrangian for the closed bradyonic bosonic string. In section 5 we obtain a expression for the Lagrangian of a closed tachyonic bosonic string. In section 6 we give a expression for the propagator of the string (do not confuse with the string field propagator of section 9). In section 7 we give a new representation for the states of the string using CUET of compact support. In section 8 we give expressions for the field of the string, the 
string field propagator and the creation and anihilation operators of a string - In section 9, we give expressions for the non-local action of a free string and a non-local interaction lagrangian for the string field similar to $\lambda \phi^{4}$ in Quantum Field Theory. Also we show how to evaluate the convolution of two string field propagators. Finally, section 10 is reserved for a discussion of the principal results.

\section{Ultradistributions of Exponential Type}

Let $\mathcal{S}$ be the Schwartz space of rapidly decreasing test functions. Let $\Lambda_{j}$ be the region of the complex plane defined as:

$$
\Lambda_{j}=\{z \in \mathbb{C}:|\mathfrak{I}(z)|<j: j \in \mathbb{N}\}
$$

According to ref. [6, 8] be the space of test functions $\hat{\phi} \in \mathrm{V}_{j}$ is constituted by all entire analytic functions of $\mathcal{S}$ for which

$$
\|\hat{\phi}\|_{j}=\max _{k \leq j}\left\{\sup _{z \in \Lambda_{j}}\left[e^{(j|\Re(z)|)}\left|\hat{\phi}^{(k)}(z)\right|\right]\right\}
$$

is finite.

The space $\mathbf{Z}$ is then defined as:

$$
\mathrm{Z}=\bigcap_{j=0}^{\infty} \mathrm{V}_{j}
$$


It is a complete countably normed space with the topology generated by the system of semi-norms $\left\{\|\cdot\|_{j}\right\}_{j \in \mathbb{N}}$. The dual of $\mathbf{Z}$, denoted by $B$, is by definition the space of ultradistributions of exponential type (ref. [6, 8]). Let $S$ the space of rapidly decreasing sequences. According to ref.[12] $S$ is a nuclear space. We consider now the space of sequences $\mathrm{P}$ generated by the Taylor development of $\hat{\phi} \in Z$

$$
P=\left\{\mathrm{Q}: \mathrm{Q}\left(\hat{\phi}(0), \hat{\phi}^{\prime}(0), \frac{\hat{\phi}^{\prime \prime}(0)}{2}, \ldots, \frac{\hat{\phi}^{(\mathrm{n})}(0)}{n !}, \ldots\right): \hat{\phi} \in \mathrm{Z}\right\}
$$

The norms that define the topology of $\mathrm{P}$ are given by:

$$
\|\hat{\phi}\|_{p}^{\prime}=\sup _{n} \frac{n^{p}}{n}\left|\hat{\phi}^{n}(0)\right|
$$

$\mathrm{P}$ is a subespace of $\mathrm{S}$ and therefore is a nuclear space. As the norms $\|\cdot\|_{j}$ and $\|\cdot\|_{p}^{\prime}$ are equivalent, the correspondence

$$
\mathrm{Z} \Longleftrightarrow \mathrm{P}
$$

is an isomorphism and therefore $\mathbf{Z}$ is a countably normed nuclear space. We can define now the set of scalar products

$$
\begin{gathered}
<\hat{\phi}(z), \hat{\psi}(z)>_{n}=\sum_{q=0}^{n} \int_{-\infty}^{\infty} e^{2 n|z|} \overline{\hat{\phi}^{(q)}}(z) \hat{\psi}^{(q)}(z) d z= \\
\sum_{q=0}^{n} \int_{-\infty}^{\infty} e^{2 n|x|} \overline{\hat{\phi}^{(q)}}(x) \hat{\psi}^{(q)}(x) d x
\end{gathered}
$$


This scalar product induces the norm

$$
\|\hat{\phi}\|_{n}^{\prime \prime}=\left[<\hat{\phi}(x), \hat{\phi}(x)>_{n}\right]^{\frac{1}{2}}
$$

The norms $\|\cdot\|_{j}$ and $\|\cdot\|_{n}^{\prime \prime}$ are equivalent, and therefore $\mathbf{Z}$ is a countably hilbertian nuclear space. Thus, if we call now $Z_{p}$ the completion of $Z$ by the norm $p$ given in (2.8), we have:

$$
\mathrm{Z}=\bigcap_{\mathrm{p}=0}^{\infty} \mathrm{Z}_{\mathrm{p}}
$$

where

$$
\mathrm{Z}_{0}=\mathrm{H}
$$

is the Hilbert space of square integrable functions.

As a consequence the "nested space"

$$
\mathrm{U}=(\mathrm{Z}, \mathrm{H}, \mathrm{B})
$$

is a Guelfand's triplet (or a Rigged Hilbert space=RHS. See ref.[12]).

Any Guelfand's triplet $\mathbf{G}=\left(\boldsymbol{\Phi}, \mathbf{H}, \mathbf{\Phi}^{\prime}\right)$ has the fundamental property that a linear and symmetric operator on $\boldsymbol{\Phi}$, admitting an extension to a self-adjoint operator in $\mathbf{H}$, has a complete set of generalized eigen-functions in $\boldsymbol{\Phi}^{\prime}$ with real eigenvalues. 
$B$ can also be characterized in the following way ( refs. [6], [8] ): let $E_{\omega}$ be the space of all functions $\hat{F}(z)$ such that:

I- $\hat{F}(z)$ is analytic for $\{z \in \mathbb{C}:|\operatorname{Im}(z)|>p\}$.

II- $\hat{\mathrm{F}}(z) e^{-p|\Re(z)|} / z^{\mathfrak{p}}$ is bounded continuous in $\{z \in \mathbb{C}:|\operatorname{Im}(z)| \geqq p\}$, where $p=0,1,2, \ldots$ depends on $\hat{F}(z)$.

Let $N$ be: $N=\left\{\hat{F}(z) \in E_{\omega}: \hat{F}(z)\right.$ is entire analytic $\}$. Then $B$ is the quotient space:

\section{III- $B=E_{\omega} / N$}

Due to these properties it is possible to represent any ultradistribution as ( ref.[6, 8] ):

$$
\hat{\mathrm{F}}(\hat{\phi})=<\hat{\mathrm{F}}(z), \hat{\phi}(z)>=\oint_{\Gamma} \hat{\mathrm{F}}(z) \hat{\phi}(z) d z
$$

where the path $\Gamma_{j}$ runs parallel to the real axis from $-\infty$ to $\infty$ for $\operatorname{Im}(z)>\zeta$, $\zeta>p$ and back from $\infty$ to $-\infty$ for $\operatorname{Im}(z)<-\zeta,-\zeta<-p$. ( $\Gamma$ surrounds all the singularities of $\hat{\mathrm{F}}(z))$.

Formula (2.12) will be our fundamental representation for a tempered ultradistribution. Sometimes use will be made of "Dirac formula" for exponential ultradistributions ( ref.[6] ):

$$
\hat{\mathrm{F}}(z) \equiv \frac{1}{2 \pi i} \int_{-\infty}^{\infty} \frac{\hat{\mathrm{f}}(\mathrm{t})}{\mathrm{t}-z} \mathrm{dt} \equiv \frac{\cosh (\lambda z)}{2 \pi i} \int_{-\infty}^{\infty} \frac{\hat{\mathrm{f}}(\mathrm{t})}{(\mathrm{t}-z) \cosh (\lambda t)} d t
$$


where the "density" $\hat{f}(t)$ is such that

$$
\oint_{\Gamma} \hat{\mathrm{F}}(z) \hat{\phi}(z) \mathrm{d} z=\int_{-\infty}^{\infty} \hat{f}(\mathrm{t}) \hat{\phi}(\mathrm{t}) \mathrm{dt}
$$

(2.13) should be used carefully. While $\hat{F}(z)$ is analytic on $\Gamma$, the density $\hat{f}(t)$ is in general singular, so that the r.h.s. of (2.14) should be interpreted in the sense of distribution theory.

Another important property of the analytic representation is the fact that on $\Gamma, \hat{F}(z)$ is bounded by a exponential and a power of $z$ (ref. [6, 8] ):

$$
|\hat{\mathrm{F}}(z)| \leq \mathrm{C}|z|^{\mathrm{p}} e^{\mathrm{p}|\mathfrak{R}(z)|}
$$

where $C$ and $p$ depend on $\hat{F}$.

The representation (2.12) implies that the addition of any entire function $\hat{\mathrm{G}}(z) \in \mathrm{N}$ to $\hat{\mathrm{F}}(z)$ does not alter the ultradistribution:

$$
\oint_{\Gamma}\{\hat{F}(z)+\hat{G}(z)\} \hat{\phi}(z) d z=\oint_{\Gamma} \hat{F}(z) \hat{\phi}(z) d z+\oint_{\Gamma} \hat{G}(z) \hat{\phi}(z) d z
$$

But:

$$
\oint_{\Gamma} \hat{\mathrm{G}}(z) \hat{\phi}(z) \mathrm{d} z=0
$$

as $\hat{\mathrm{G}}(z) \hat{\phi}(z)$ is entire analytic ( and rapidly decreasing ),

$$
\therefore \oint_{\Gamma}\{\hat{\mathrm{F}}(z)+\hat{\mathrm{G}}(z)\} \hat{\phi}(z) \mathrm{d} z=\oint_{\Gamma} \hat{\mathrm{F}}(z) \hat{\phi}(z) \mathrm{d} z
$$


Another very important property of B is that B is reflexive under the Fourier transform:

$$
\mathrm{B}=\mathcal{F}_{\mathrm{c}}\{\mathrm{B}\}=\mathcal{F}\{\mathrm{B}\}
$$

where the complex Fourier transform $F(k)$ of $\hat{F}(z) \in B$ is given by:

$$
\begin{gathered}
F(k)=\Theta[\mathfrak{I}(k)] \int_{\Gamma_{+}} \hat{\mathrm{F}}(z) e^{i k z} \mathrm{~d} z-\Theta[-\Im(k)] \int_{\Gamma_{-}} \hat{\mathrm{F}}(z) e^{i k z} \mathrm{~d} z= \\
\Theta[\Im(k)] \int_{0}^{\infty} \hat{\mathrm{f}}(\mathrm{x}) e^{i k x} \mathrm{~d} x-\Theta[-\Im(k)] \int_{-\infty}^{0} \hat{\mathrm{f}}(x) e^{i k x} \mathrm{~d} x
\end{gathered}
$$

Here $\Gamma_{+}$is the part of $\Gamma$ with $\mathfrak{R}(z) \geq 0$ and $\Gamma_{-}$is the part of $\Gamma$ with $\mathfrak{R}(z) \leq 0$ Using (2.18) we can interpret Dirac's formula as:

$$
\mathrm{F}(\mathrm{k}) \equiv \frac{1}{2 \pi \mathrm{i}} \int_{-\infty}^{\infty} \frac{\mathrm{f}(\mathrm{s})}{\mathrm{s}-\mathrm{k}} \mathrm{ds} \equiv \mathcal{F}_{\mathrm{c}}\left\{\mathcal{F}^{-1}\{\mathrm{f}(\mathrm{s})\}\right\}
$$

The treatment for ultradistributions of exponential type defined on $\mathbb{C}^{n}$ is similar to the case of one variable. Thus

$$
\begin{array}{r}
\Lambda_{j}=\left\{z=\left(z_{1}, z_{2}, \ldots, z_{\mathfrak{n}}\right) \in \mathbb{C}^{\mathfrak{n}}:\left|\mathfrak{I}\left(z_{k}\right)\right| \leq j \quad 1 \leq k \leq \mathfrak{n}\right\} \\
\|\hat{\phi}\|_{j}=\max _{k \leq j}\left\{\sup _{z \in \Lambda_{j}}\left[e^{j\left[\sum_{p=1}^{n}\left|\Re\left(z_{p}\right)\right|\right]}\left|D^{(k)} \hat{\phi}(z)\right|\right]\right\}
\end{array}
$$

where $D^{(k)}=\partial^{\left(k_{1}\right)} \partial^{\left(k_{2}\right)} \cdots \partial^{\left(k_{n}\right)} \quad k=k_{1}+k_{2}+\cdots+k_{n}$

$B^{n}$ is characterized as follows. Let $E_{\omega}^{n}$ be the space of all functions $\hat{F}(z)$ such that: 
$\mathbf{I}^{\prime}-\hat{\mathrm{F}}(z)$ is analytic for $\left\{z \in \mathbb{C}^{\mathfrak{n}}:\left|\operatorname{Im}\left(z_{1}\right)\right|>p,\left|\operatorname{Im}\left(z_{2}\right)\right|>p, \ldots,\left|\operatorname{Im}\left(z_{n}\right)\right|>\right.$ p\}.

$\mathbf{I I}^{\prime}-\hat{\mathrm{F}}(z) e^{-\left[\mathfrak{p} \sum_{j=1}^{n}\left|\Re\left(z_{j}\right)\right|\right]} / z^{\mathfrak{p}}$ is bounded continuous in $\left\{z \in \mathbb{C}^{\mathfrak{n}}:\left|\operatorname{Im}\left(z_{1}\right)\right| \geqq\right.$ $\left.p,\left|\operatorname{Im}\left(z_{2}\right)\right| \geqq p, \ldots,\left|\operatorname{Im}\left(z_{n}\right)\right| \geqq p\right\}$, where $p=0,1,2, \ldots$ depends on $\hat{F}(z)$.

Let $N^{n}$ be: $N^{n}=\left\{\hat{F}(z) \in E_{\omega}^{n}: \vec{F}(z)\right.$ is entire analytic at minus in one of the variables $\left.z_{j} \quad 1 \leq j \leq n\right\}$ Then $B^{n}$ is the quotient space:

III $^{\prime}-\mathrm{B}^{\mathrm{n}}=\mathrm{E}_{\omega}^{\mathrm{n}} / \mathrm{N}^{\mathrm{n}}$ We have now

$$
\hat{\mathrm{F}}(\hat{\phi})=<\hat{\mathrm{F}}(z), \hat{\phi}(z)>=\oint_{\Gamma} \hat{\mathrm{F}}(z) \hat{\phi}(z) \mathrm{d} z_{1} \mathrm{~d} z_{2} \cdots \mathrm{d} z_{\mathrm{n}}
$$

$\Gamma=\Gamma_{1} \cup \Gamma_{2} \cup \ldots \Gamma_{\mathrm{n}}$ where the path $\Gamma_{\mathrm{j}}$ runs parallel to the real axis from $-\infty$ to $\infty$ for $\operatorname{Im}\left(z_{\mathfrak{j}}\right)>\zeta, \zeta>p$ and back from $\infty$ to $-\infty$ for $\operatorname{Im}\left(z_{\mathfrak{j}}\right)<-\zeta,-\zeta<-p$. (Again $\Gamma$ surrounds all the singularities of $\hat{F}(z)$ ). The n-dimensional Dirac's formula is

$$
\hat{\mathrm{F}}(z)=\frac{1}{(2 \pi i)^{n}} \int_{-\infty}^{\infty} \frac{\hat{f}(t)}{\left(t_{1}-z_{1}\right)\left(t_{2}-z_{2}\right) \ldots\left(t_{n}-z_{n}\right)} d t_{1} d t_{2} \ldots d t_{n}
$$

where the "density" $\hat{f}(t)$ is such that

$$
\oint_{\Gamma} \hat{F}(z) \hat{\phi}(z) d z_{1} d z_{2} \cdots d z_{n}=\int_{-\infty}^{\infty} f(t) \hat{\phi}(t) d t_{1} d t_{2} \cdots d t_{n}
$$

and the modulus of $\hat{F}(z)$ is bounded by

$$
|\hat{\mathrm{F}}(z)| \leq \mathrm{C}|z|^{\mathrm{p}} e^{\left[p_{j=1}^{n} \sum_{j=1}^{n}\left(z_{j}\right) \mid\right]}
$$


where $C$ and $p$ depend on $\hat{F}$.

\section{The Case $\mathrm{N} \rightarrow \infty$}

When the number of variables of the argument of the Ultradistribution of Exponential type tends to infinity we define:

$$
d \mu(x)=\frac{e^{-x^{2}}}{\sqrt{\pi}} d x
$$

Let $\hat{\phi}\left(x_{1}, x_{2}, \ldots, x_{n}\right)$ be such that:

$$
\int_{-\infty}^{\infty} \ldots \int\left|\hat{\phi}\left(x_{1}, x_{2}, \ldots, x_{n}\right)\right|^{2} d \mu_{1} d \mu_{2} \ldots d \mu_{n}<\infty
$$

where

$$
d \mu_{i}=\frac{e^{-x_{i}^{2}}}{\sqrt{\pi}} d x_{i}
$$

Then by definition $\hat{\phi}\left(x_{1}, x_{2}, \ldots, x_{n}\right) \in L_{2}\left(\mathbb{R}^{n}, \mu\right)$ and

$$
\mathrm{L}_{2}\left(\mathbb{R}^{\infty}, \mu\right)=\bigcup_{n=1}^{\infty} \mathrm{L}_{2}\left(\mathbb{R}^{\mathrm{n}}, \mu\right)
$$

Let $\hat{\psi}$ be givem by

$$
\hat{\psi}\left(z_{1}, z_{2}, \ldots, z_{n}\right)=\pi^{n / 4} \hat{\phi}\left(z_{1}, z_{2}, \ldots, z_{n}\right) e^{\frac{z_{1}^{2}+z_{2}^{2}+\ldots+z_{n}^{2}}{2}}
$$


where $\hat{\phi} \in Z^{n}$ (the corresponding n-dimensional of $Z$ ).

Then by definition $\hat{\psi}\left(z_{1}, z_{2}, \ldots, z_{n}\right) \in \mathrm{G}\left(\mathbb{C}^{n}\right)$,

$$
\mathrm{G}\left(\mathbb{C}^{\infty}\right)=\bigcup_{n=1}^{\infty} \mathrm{G}\left(\mathbb{C}^{n}\right)
$$

and the dual $\mathrm{G}^{\prime}\left(\mathbb{C}^{\infty}\right)$ given by

$$
G^{\prime}\left(\mathbb{C}^{\infty}\right)=\bigcup_{n=1}^{\infty} G^{\prime}\left(\mathbb{C}^{n}\right)
$$

is the space of Ultradistributions of Exponential type.

The analog to (2.11) in the infinite dimensional case is:

$$
W=\left(G\left(\mathbb{C}^{\infty}\right), L_{2}\left(\mathbb{R}^{\infty}, \mu\right), G^{\prime}\left(\mathbb{C}^{\infty}\right)\right)
$$

If we define:

$$
\mathcal{F}: \mathrm{G}\left(\mathbb{C}^{\infty}\right) \rightarrow \mathrm{G}\left(\mathbb{C}^{\infty}\right)
$$

via the Fourier transform:

$$
\mathcal{F}: \mathrm{G}\left(\mathbb{C}^{\mathrm{n}}\right) \rightarrow \mathrm{G}\left(\mathbb{C}^{\mathrm{n}}\right)
$$

given by:

$$
\mathcal{F}\{\hat{\psi}\}(k)=\int_{-\infty}^{\infty} \hat{\psi}\left(z_{1}, z_{2}, \ldots, z_{n}\right) e^{i k \cdot z+\frac{k^{2}}{2}} d \rho_{1} d \rho_{2} \ldots d \rho_{n}
$$


where

$$
\mathrm{d} \rho(z)=\frac{e^{-\frac{z^{2}}{2}}}{\sqrt{2 \pi}} d z
$$

we conclude that

$$
\mathrm{G}^{\prime}\left(\mathbb{C}^{\infty}\right)=\mathcal{F}_{\mathrm{c}}\left\{\mathrm{G}^{\prime}\left(\mathbb{C}^{\infty}\right)\right\}=\mathcal{F}\left\{\mathrm{G}^{\prime}\left(\mathbb{C}^{\infty}\right)\right\}
$$

where in the one-dimensional case

$$
\mathcal{F}_{c}\{\hat{\psi}\}(k)=\Theta[\mathfrak{I}(k)] \int_{\Gamma_{+}} \hat{\psi}(z) e^{i k z+\frac{k^{2}}{2}} \mathrm{~d} \rho-\Theta[-\mathfrak{I}(\mathrm{k})] \int_{\Gamma_{-}} \hat{\psi}(z) e^{i k z+\frac{k^{2}}{2}} \mathrm{~d} \rho
$$

\section{The Constraints for a Bradyonic Bosonic String}

As is known the Nambu-Goto Lagrangian for the bosonic string is given by $([13])$

$$
\mathcal{L}_{\mathrm{NG}}=\mathrm{T} \sqrt{\left(\dot{\mathrm{X}} \cdot \mathrm{X}^{\prime}\right)^{2}-\dot{\mathrm{X}}^{2} \mathrm{X}^{\prime 2}}
$$

where

$$
X=X(\tau, \sigma) ; \dot{X}=\partial_{\tau} X ; X^{\prime}=\partial_{\sigma} X
$$

If we use the constraint

$$
\left(\dot{X}-X^{\prime}\right)^{2}=0
$$


we obtain:

$$
\dot{X}^{4}+X^{\prime 4}=4\left(\dot{X} \cdot X^{\prime}\right)^{2}-2 \dot{X}^{2} X^{\prime 2} \geq 0
$$

On the other hand

$$
\left(\dot{X}^{2}-X^{\prime 2}\right)^{2}=\dot{X}^{4}+X^{\prime 4}-2 \dot{X}^{2} X^{\prime 2}
$$

and from (4.3) we have

$$
4 \mathcal{L}_{\mathrm{BS}}^{2}=\mathrm{T}^{2}\left(\dot{\mathrm{X}}^{2}-\mathrm{X}^{\prime 2}\right)^{2}=4 \mathrm{~T}^{2}\left[\left(\dot{\mathrm{X}} \cdot \mathrm{X}^{\prime}\right)^{2}-\dot{\mathrm{X}}^{2} \mathrm{X}^{\prime 2}\right]=4 \mathcal{L}_{\mathrm{NG}}^{2} \geq 0
$$

As a consequence of (4.5):

$$
\mathcal{L}_{\mathrm{NG}}=\mathrm{T} \sqrt{\left(\dot{\mathrm{X}} \cdot \mathrm{X}^{\prime}\right)^{2}-\dot{\mathrm{X}}^{2} \mathrm{X}^{\prime 2}}=\frac{\mathrm{T}}{2}\left|\dot{\mathrm{X}}^{2}-\mathrm{X}^{\prime 2}\right|=\mathcal{L}_{\mathrm{BS}}
$$

We then see that is sufficient to use only one constraint to obtain the Lagrangian for a bosonic string theory from the Nambú-Goto Lagrangian. Another constraint from which (4.6) follows is

$$
\left(\dot{X}+X^{\prime}\right)^{2}=0
$$

Thus, the equations for the bosonic string reduce to:

$$
\left\{\begin{array}{l}
\ddot{X}-X^{\prime \prime}=0 \\
\left(\dot{X}+X^{\prime}\right)^{2}=0 \\
X_{\mu}(\tau, 0)=X_{\mu}(\tau, \pi)=0
\end{array}\right.
$$


or equivalently

$$
\left\{\begin{array}{l}
\ddot{X}-X^{\prime \prime}=0 \\
\left(\dot{X}-X^{\prime}\right)^{2}=0 \\
X_{\mu}(\tau, 0)=X_{\mu}(\tau, \pi)=0
\end{array}\right.
$$

The Euler-Lagrange equations for (4.8) and (4.9) are respectively:

$$
\begin{gathered}
4 \delta\left(\dot{X}^{2}-X^{\prime 2}\right)\left[\left(\dot{X} \cdot \ddot{X}-X^{\prime} \cdot \dot{X}^{\prime}\right) \dot{X}_{\mu}-\left(X^{\prime} \cdot \dot{X}^{\prime}-X^{\prime} \cdot X^{\prime \prime}\right) X_{\mu}^{\prime}\right]+ \\
\operatorname{Sgn}\left(\dot{X}^{2}-X^{\prime 2}\right)\left(\ddot{X}-X^{\prime \prime}\right)+\lambda\left(\ddot{X}+2 \dot{X}^{\prime}+X^{\prime \prime}\right)=0 \\
4 \delta\left(\dot{X}^{2}-X^{\prime 2}\right)\left[\left(\dot{X} \cdot \ddot{X}-X^{\prime} \cdot \dot{X}^{\prime}\right) \dot{X}_{\mu}-\left(X^{\prime} \cdot \dot{X}^{\prime}-X^{\prime} \cdot X^{\prime \prime}\right) X_{\mu}^{\prime}\right]+ \\
\operatorname{Sgn}\left(\dot{X}^{2}-X^{\prime 2}\right)\left(\ddot{X}-X^{\prime \prime}\right)+\lambda\left(\ddot{X}-2 \dot{X}^{\prime}+X^{\prime \prime}\right)=0
\end{gathered}
$$

where $\lambda$ is a Lagrange multiplier.

If we define a physical state of the string as:

$$
p^{2} \mid \Phi>=0
$$

A solution of (4.8) defined on physical states is:

$$
\left\{\begin{array}{l}
X_{\mu}(\tau, \sigma)=x_{\mu}+l^{2} p_{\mu} \tau+\frac{i l}{2} \sum_{n=-\infty ; n \neq 0}^{\infty} \frac{a_{n}}{n} e^{-2 i n(\tau-\sigma)} \\
p^{2} \mid \Phi>=0
\end{array}\right.
$$


It is immediate to prove that (4.13) is solution of Nambu-Goto equations on physical states, (Nambu-Goto equations arise from Euler-Lagrange equations corresponding to the Lagrangian (4.1), and it is easy to prove that the currently used solution for the closed string movement is not solution of Nambu-Goto equations due to the fact that Virasoro operators $L_{n}$ and $\tilde{L}_{n}$ does not anihilate the physical states for $n<0$ ) and moreover, does not form a set of commuting operators.

In a similar way a solution of (4.9) at the is:

$$
\left\{\begin{array}{l}
X_{\mu}(\tau, \sigma)=x_{\mu}+l^{2} p_{\mu} \tau+\frac{i l}{2} \sum_{n=-\infty ; n \neq 0}^{\infty} \frac{\tilde{a}_{n}}{n} e^{-2 i n(\tau+\sigma)} \\
p^{2} \mid \Phi>=0
\end{array}\right.
$$

\section{The constraints for a Tachyonic Bosonic String}

The Nambu-Goto Lagrangian for the tachyonic bosonic string is given by

$$
\mathcal{L}_{\mathrm{NG}}=\mathrm{T} \sqrt{\dot{\mathrm{X}}^{2} \mathrm{X}^{\prime 2}-\left(\dot{\mathrm{X}} \cdot \mathrm{X}^{\prime}\right)^{2}}
$$

If we use the constraint

$$
\left(\dot{X} \pm i X^{\prime}\right)^{2}=0
$$


we obtain

$$
\dot{X}^{4}+X^{\prime} 4=2 \dot{X}^{2} X^{\prime 2}-4\left(\dot{X} \cdot X^{\prime}\right)^{2} \geq 0
$$

On the other hand

$$
\left(\dot{X}^{2}+X^{\prime 2}\right)^{2}=\dot{X}^{4}+X^{\prime 4}+2 \dot{X}^{2} X^{\prime 2} \geq 0
$$

and from (5.3) we have

$$
4 \mathcal{L}_{\mathrm{BS}}^{2}=\mathrm{T}^{2}\left(\dot{\mathrm{X}}^{2}+\mathrm{X}^{\prime 2}\right)^{2}=4 \mathrm{~T}^{2}\left[\dot{\mathrm{X}}^{2} \mathrm{X}^{\prime 2}-\left(\dot{\mathrm{X}} \cdot \mathrm{X}^{\prime}\right)^{2}\right]=4 \mathcal{L}_{\mathrm{NG}}^{2} \geq 0
$$

As a consequence of (5.5):

$$
\mathcal{L}_{\mathrm{NG}}=\mathrm{T} \sqrt{\dot{\mathrm{X}}^{2} \mathrm{X}^{\prime 2}-\left(\dot{X} \cdot \mathrm{X}^{\prime}\right)^{2}}=\frac{\mathrm{T}}{2}\left|\dot{\mathrm{X}}^{2}+\mathrm{X}^{\prime 2}\right|=\mathcal{L}_{\mathrm{BS}}
$$

\section{The propagator of the closed bosonic string}

We write $X_{\mu}$ in (4.13) as:

$$
X_{\mu}(\tau, \sigma)=x_{\mu}+l^{2} p_{\mu} \tau+l \int_{-\infty}^{\infty}\left(\frac{a_{\mu}(k)}{\sqrt{2|k|}} e^{-i(|k| \tau-k \sigma)}+\frac{a_{\mu}^{+}(k)}{\sqrt{2|k|}} e^{i(|k| \tau-k \sigma)}\right) d k
$$

where:

$$
\begin{aligned}
a_{\mu}(k) & =i \sum_{n>0} a_{\mu n} \delta(k-2 n) \\
a_{\mu}^{+}(k) & =-i \sum_{n>0} a_{\mu n}^{+} \delta(k-2 n)
\end{aligned}
$$


with:

$$
\left[a_{\mu m}, a_{v n}^{+}\right]=\eta_{\mu \nu} \delta_{m n}
$$

and

$$
\begin{gathered}
{\left[a_{\mu}(k), a_{v}^{+}\left(k^{\prime}\right)\right]=\eta_{\mu \nu} \delta\left(k-k^{\prime}\right) \sum_{n>0} \delta(k-2 n)=} \\
\frac{\eta_{\mu v}}{4} \Theta(k) \delta\left(k-k^{\prime}\right) \sum_{n=-\infty}^{\infty} e^{-i n \pi k}
\end{gathered}
$$

With the usual definition

$$
\Delta_{\mu \nu}\left(\tau-\tau^{\prime}, \sigma-\sigma^{\prime}\right)=<0\left|T\left[X_{\mu}(\tau, \sigma) X_{v}\left(\tau^{\prime}, \sigma^{\prime}\right)\right]\right| 0>
$$

the propagator for the string is (do not confuse with the string field propagator of section 9):

$$
\begin{gathered}
\Delta_{\mu \nu}\left(\tau-\tau^{\prime}, \sigma-\sigma^{\prime}\right)= \\
\eta_{\mu \nu} \frac{l^{2}}{4} \sum_{n>0} n^{-1} e^{-2 \operatorname{insgn}\left(\tau-\tau^{\prime}\right)\left[\left(\tau-\tau^{\prime}\right)-\left(\sigma-\sigma^{\prime}\right)\right]} \operatorname{Sgn}\left(\tau-\tau^{\prime}\right)
\end{gathered}
$$

For $X_{\mu}$ in (4.14) we obtain:

$$
\begin{gathered}
\Delta_{\mu \nu}\left(\tau-\tau^{\prime}, \sigma-\sigma^{\prime}\right)= \\
\eta_{\mu \nu} \frac{l^{2}}{4} \sum_{n>0} n^{-1} e^{-2 \operatorname{ing} \operatorname{Sgn}\left(\tau-\tau^{\prime}\right)\left[\left(\tau-\tau^{\prime}\right)+\left(\sigma-\sigma^{\prime}\right)\right]} \operatorname{Sgn}\left(\tau-\tau^{\prime}\right)
\end{gathered}
$$




\section{A representation of the states of the closed}

\section{bosonic string}

\section{The case $\mathbf{n}$ finite}

For an ultradistribution of exponential type, we can write:

$$
\begin{array}{r}
\mathrm{G}(\mathrm{k})=\oint_{\Gamma_{z}}\{\Theta[\mathfrak{I}(\mathrm{k})] \Theta[\mathfrak{R}(z)]-\Theta[-\mathfrak{I}(\mathrm{k})] \Theta[-\mathfrak{R}(z)]\} \hat{\mathrm{G}}(z) e^{i k z} \mathrm{~d} z \\
\hat{\mathrm{G}}(z)=\oint_{\Gamma_{\mathrm{k}}}\{\Theta[\mathfrak{I}(z)] \Theta[-\mathfrak{R}(\mathrm{k})]-\Theta[-\mathfrak{I}(z)] \Theta[\mathfrak{R}(\mathrm{k})]\} \hat{\mathrm{G}}(z) e^{-i k z} \mathrm{dk}
\end{array}
$$

and

$$
\begin{gathered}
\mathrm{G}(\phi)=\oint_{\Gamma_{\mathrm{k}}} \mathrm{G}(\mathrm{k}) \phi(\mathrm{k}) \mathrm{dk}= \\
\oint_{\Gamma_{\mathrm{k}}} \oint_{\Gamma_{z}}\{\Theta[\mathfrak{I}(\mathrm{k})] \Theta[\mathfrak{R}(z)]-\Theta[-\mathfrak{I}(\mathrm{k})] \Theta[-\mathfrak{R}(z)]\} \hat{\mathrm{G}}(z) \phi(k) e^{i k z} \mathrm{dk} d z= \\
-i \oint_{\Gamma_{\mathrm{k}}} \oint_{\Gamma_{z}^{\prime}}\{\Theta[\mathfrak{I}(k)] \Theta[\mathfrak{I}(z)]-\Theta[-\mathfrak{I}(\mathrm{k})] \Theta[-\mathfrak{I}(z)]\} \hat{\mathrm{G}}(-\mathrm{i} z) \phi(k) e^{\mathrm{k} z} \mathrm{dk} d z
\end{gathered}
$$

where the path $\Gamma_{z}^{\prime}$ is the path $\Gamma_{z}$ rotated ninety degrees counterclockwise around the origin of the complex plane.

If $F(z)$ is an UET of compact support we can define:

$$
<\hat{\mathrm{F}}(z), \phi(z)>=
$$




$$
\oint_{\Gamma_{\mathrm{k}}} \oint_{\Gamma_{z}^{\prime}}\{\Theta[\Im(\mathrm{I})] \Theta[\Im(z)]-\Theta[-\mathfrak{I}(\mathrm{k})] \Theta[-\Im(z)]\} \hat{\mathrm{F}}(z) \phi(\mathrm{k}) e^{\mathrm{k} z} \mathrm{dk} \mathrm{d} z
$$

then:

$$
\begin{aligned}
& <\hat{\mathrm{F}}^{\prime}(z), \phi(z)>= \\
& \oint_{\Gamma_{\mathrm{k}}} \oint_{\Gamma_{z}^{\prime}}\{\Theta[\Im(k)] \Theta[\mathfrak{I}(z)]-\Theta[-\mathfrak{I}(\mathrm{k})] \Theta[-\Im(z)]\} \hat{\mathrm{F}}^{\prime}(z) \phi(k) e^{\mathrm{k} z} \mathrm{dk} \mathrm{d} z= \\
& -\oint_{\Gamma_{\mathrm{k}}} \oint_{\Gamma_{z}^{\prime}}\{\Theta[\Im(\mathrm{I})] \Theta[\Im(z)]-\Theta[-\Im(k)] \Theta[-\Im(z)]\} \hat{\mathrm{F}}(z) \mathrm{k} \phi(\mathrm{k}) e^{\mathrm{k} z} \mathrm{dk} \mathrm{d} z= \\
& <\hat{\mathrm{F}}(z),-z \phi(z)>
\end{aligned}
$$

If we define:

$$
a=-z \quad ; \quad a^{+}=\frac{d}{d z}
$$

we have

$$
\left[\mathrm{a}, \mathrm{a}^{+}\right]=1
$$

Thus we have a representation for creation and annihilation operators of the states of the string. The vacuum state annihilated by $z_{\mu}$ is the UET $\delta\left(z_{\mu}\right)$, and the orthonormalized states obtained by sucessive application of $\frac{\mathrm{d}}{\mathrm{d} z_{\mu}}$ to $\delta\left(z_{\mu}\right)$ are:

$$
F_{n}\left(z_{\mu}\right)=\frac{\delta^{(n)}\left(z_{\mu}\right)}{\sqrt{n !}}
$$

On the real axis:

$$
<\hat{\mathrm{F}}(z), \phi(z)>=\int_{-\infty}^{\infty} \int_{-\infty}^{\infty} \hat{\mathrm{f}}(x) \phi(k) e^{k x} d x d k
$$


where $\bar{f}(x)$ is given by Dirac's formula:

$$
\hat{\mathrm{F}}(z)=\frac{1}{2 \pi i} \int_{-\infty}^{\infty} \frac{\overline{\hat{f}}(x)}{x-z} d x
$$

A general state of the string can be writen as:

$$
\begin{gathered}
\phi(x,\{z\})=\left[a_{0}(x)+a_{\mu_{1}}^{i_{1}}(x) \partial_{i_{1}}^{\mu_{1}}+a_{\mu_{1} \mu_{2}}^{i_{1} i_{2}}(x) \partial_{i_{1}}^{\mu_{1}} \partial_{i_{2}}^{\mu_{2}}+\ldots+\ldots\right. \\
\left.+a_{\mu_{1} \mu_{2} \ldots \mu_{n}}^{i_{1} i_{2} \ldots i_{n}}(x) \partial_{i_{1}}^{\mu_{1}} \partial_{1_{2}}^{\mu_{2}} \ldots \partial_{i_{n}}^{\mu_{n}}+\ldots+\ldots\right] \delta(\{z\})
\end{gathered}
$$

where $\{z\}$ denotes $\left(z_{1 \mu}, z_{2 \mu}, \ldots, z_{n \mu}, \ldots, \ldots.\right)$, and $\phi$ is a UET of compact support in the set of variables $\{z\}$. The functions $a_{\mu_{1} \mu_{2} \ldots \mu_{n}}^{i_{1} i_{2} \ldots i_{n}}(x)$ are solutions of

$$
\square \mathrm{a}_{\mu_{1} \mu_{2} \ldots \mu_{n}}^{i_{1} i_{2} \ldots i_{n}}(x)=0
$$

\section{The case $\mathbf{n} \rightarrow \infty$}

In this case

$$
\begin{gathered}
\mathrm{G}(\mathrm{k})=\oint_{\Gamma_{z}}\{\Theta[\mathfrak{I}(\mathrm{k})] \Theta[\mathfrak{R}(z)]-\Theta[-\mathfrak{I}(\mathrm{k})] \Theta[-\mathfrak{R}(z)]\} \hat{\mathrm{G}}(z) e^{i k z+\frac{\mathrm{k}^{2}}{2}-\frac{z^{2}}{2}} \frac{\mathrm{d} z}{\sqrt{2 \pi}} \\
\hat{\mathrm{G}}(z)=\oint_{\Gamma_{\mathrm{k}}}\{\Theta[\mathfrak{I}(z)] \Theta[-\mathfrak{R}(\mathrm{k})]-\Theta[-\mathfrak{I}(z)] \Theta[\mathfrak{R}(\mathrm{k})]\} \times \\
\hat{\mathrm{G}}(z) e^{-i k z+\frac{z^{2}}{2}-\frac{\mathrm{k}^{2}}{2}} \frac{\mathrm{dk}}{\sqrt{2 \pi}} \\
\mathrm{G}(\phi)=\oint_{\Gamma_{\mathrm{k}}} \mathrm{G}(\mathrm{k}) \phi(\mathrm{k}) \mathrm{e}^{-\mathrm{k}^{2}} \frac{\mathrm{dk}}{\sqrt{\pi}}=
\end{gathered}
$$




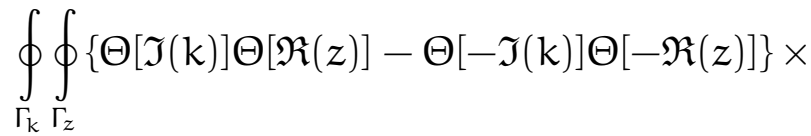

$$
\begin{aligned}
& \hat{\mathrm{G}}(z) \phi(\mathrm{k}) \mathrm{e}^{i k z-\frac{z^{2}}{2}-\mathrm{k}^{2}} \frac{\mathrm{dk} \mathrm{d} z}{\sqrt{2} \pi}= \\
& -i \oint_{\Gamma_{\mathrm{k}}} \oint_{\Gamma_{z}^{\prime}}\{\Theta[\Im(k)] \Theta[\Im(z)]-\Theta[-\Im(k)] \Theta[-\Im(z)]\} \times \\
& \hat{\mathrm{G}}(-i z) \phi(k) e^{k z+\frac{z^{2}}{2}-k^{2}} \frac{d k d z}{\sqrt{2} \pi}
\end{aligned}
$$

If $F(z)$ is an CUET we can define:

$$
\begin{aligned}
& <\hat{\mathrm{F}}(z), \phi(z)>= \\
& \oint_{\Gamma_{\mathrm{k}}} \oint_{\Gamma_{z}^{\prime}}\{\Theta[\Im(\mathrm{K})] \Theta[\Im(z)]-\Theta[-\mathfrak{I}(\mathrm{k})] \Theta[-\Im(z)]\} \times \\
& {\left[\hat{\mathrm{F}}(z) e^{-\frac{3 z^{2}}{2}}\right] \phi(k) e^{k z+\frac{z^{2}}{2}-k^{2}} \frac{d k d z}{\sqrt{2} \pi}=} \\
& \oint_{\Gamma_{\mathrm{k}}} \oint_{\Gamma_{z}^{\prime}}\{\Theta[\Im(k)] \Theta[\Im(z)]-\Theta[-\mathfrak{I}(\mathrm{k})] \Theta[-\Im(z)]\} \times \\
& \hat{\mathrm{F}}(z) \phi(k) e^{k z-z^{2}-k^{2}} \frac{d k d z}{\sqrt{2} \pi}=
\end{aligned}
$$

and then

$$
\begin{gathered}
<-2 z \hat{\mathrm{F}}(z)+\hat{\mathrm{F}}^{\prime}(z), \phi(z)>= \\
\oint_{\Gamma_{\mathrm{k}} \Gamma_{z}^{\prime}}\{\Theta[\Im(k)] \Theta[\Im(z)]-\Theta[-\Im(k)] \Theta[-\Im(z)]\} \times \\
{\left[-2 z \hat{\mathrm{F}}(z)+\hat{\mathrm{F}}^{\prime}(z)\right] \phi(k) e^{k z-z^{2}-k^{2}} \frac{\mathrm{dk} d z}{\sqrt{2} \pi}=}
\end{gathered}
$$




$$
\begin{gathered}
-\oint_{\Gamma_{\mathrm{k}}} \oint_{\Gamma_{z}^{\prime}}\{\Theta[\Im(k)] \Theta[\Im(z)]-\Theta[-\Im(k)] \Theta[-\Im(z)]\} \times \\
\hat{\mathrm{F}}(z) \mathrm{k} \phi(\mathrm{k}) \mathrm{e}^{\mathrm{k} z-z^{2}-\mathrm{k}^{2}} \frac{\mathrm{dk} \mathrm{d} z}{\sqrt{2} \pi}= \\
<\hat{\mathrm{F}}(z),-z \phi(z)>
\end{gathered}
$$

If we define:

$$
a=-z \quad ; \quad a^{+}=-2 z+\frac{d}{d z}
$$

we have

$$
\left[\mathrm{a}, \mathrm{a}^{+}\right]=1
$$

The vacuum state annihilated by a is $\delta(z) e^{z^{2}}$. The orthonormalized states obtained by sucessive application of $\mathrm{a}^{+}$are:

$$
\hat{\mathrm{F}}_{\mathrm{n}}(z)=2^{\frac{1}{4}} \pi^{\frac{1}{2}} \frac{\delta^{(\mathfrak{n})}(z) e^{z^{2}}}{\sqrt{n !}}
$$

On the real axis we have

$$
<\hat{\mathrm{F}}(z), \phi(z)>=\int_{-\infty}^{\infty} \int_{-\infty}^{\infty} \hat{\mathrm{f}}(x) \phi(k) e^{k x-x^{2}-k^{2}} \frac{\mathrm{d} x \mathrm{dk}}{\sqrt{2} \pi}
$$

where $\overline{\hat{f}}(x)$ is given by Dirac's formula:

$$
\hat{\mathrm{F}}(z)=\frac{1}{2 \pi i} \int_{-\infty}^{\infty} \frac{\overline{\hat{f}}(x)}{x-z} d x
$$




\section{The String Field}

According to (4.13) and section 7 the equation for the string field is given by

$$
\square \Phi(x,\{z\})=\left(\partial_{0}^{2}-\partial_{1}^{2}-\partial_{2}^{2}-\partial_{3}^{2}\right) \Phi(x,\{z\})=0
$$

where $\{z\}$ denotes $\left(z_{1 \mu}, z_{2 \mu}, \ldots, z_{n \mu}, \ldots, \ldots.\right)$, and $\Phi$ is a CUET in the set of variables $\{\boldsymbol{z}\}$. Any UET of compact support can be writed as a development of $\delta(\{z\})$ and its derivatives. Thus we have:

$$
\begin{aligned}
\Phi(x,\{z\}) & =\left[A_{0}(x)+A_{\mu_{1}}^{i_{1}}(x) \partial_{i_{1}}^{\mu_{1}}+A_{\mu_{1} \mu_{2}}^{i_{1} i_{2}}(x) \partial_{i_{1}}^{\mu_{1}} \partial_{i_{2}}^{\mu_{2}}+\ldots+\ldots\right. \\
& \left.+A_{\mu_{1} \mu_{2} \ldots \mu_{n}}^{i_{1} i_{2} \ldots i_{n}}(x) \partial_{i_{1}}^{\mu_{1}} \partial_{1_{2}}^{\mu_{2}} \ldots \partial_{i_{n}}^{\mu_{n}}+\ldots+\ldots\right] \delta(\{z\})
\end{aligned}
$$

where the quantum fields $A_{\mu_{1} \mu_{2} \ldots \mu_{n}}^{i_{1} i_{2} \ldots i_{n}}(x)$ are solutions of

$$
\square A_{\mu_{1} \mu_{2} \ldots \mu_{n}}^{i_{1} i_{2} \ldots i_{n}}(x)=0
$$

The propagator of the string field can be exppresed in terms of the propagators of the component fields:

$$
\begin{gathered}
\Delta\left(x-x^{\prime},\{z\},\left\{z^{\prime}\right\}\right)=\left[\Delta_{0}\left(x-x^{\prime}\right)+\Delta_{\mu_{1} \mu_{2}}^{i_{1} j_{1}}\left(x-x^{\prime}\right) \partial_{i_{1}}^{\mu_{1}} \partial_{j_{1}}^{\prime v_{1}}+\ldots+\ldots+\right. \\
\left.\Delta_{i_{1} \ldots i_{n} j_{1} \ldots j_{n}}^{\mu_{1} \ldots \mu_{n} v_{1} \ldots \nu_{n}}\left(x-x^{\prime}\right) \partial_{\mu_{1}}^{i_{1}} \ldots \partial_{\mu_{n}}^{i_{n}} \partial_{v_{1}}^{i_{1}} \ldots \partial_{v_{n}}^{\prime j_{n}}+\ldots+\ldots\right] \delta\left(\{z\},\left\{z^{\prime}\right\}\right)
\end{gathered}
$$

We define the operators of annihilation and creation of a string as:

$$
a(k,\{z\})=\left[a_{0}(k)+a_{\mu_{1}}^{i_{1}}(k) \partial_{i_{1}}^{\mu_{1}}+\ldots+\ldots+\right.
$$




$$
\begin{aligned}
& a_{\mu_{1} \ldots \mu_{n}}^{i_{1} \ldots i_{n}} \partial_{i_{1}}^{\mu_{1}} \ldots \partial_{i_{n}}^{\mu_{n}}+\ldots+\ldots . \delta(\{z\}) \\
& a^{+}\left(k,\left\{z^{\prime}\right\}\right)=\left[a_{0}^{+}(k)+a_{v_{1}}^{+j_{1}}(k) \partial_{j_{1}}^{\prime v_{1}}+\ldots+\ldots+\right. \\
& \left.a_{v_{1} \ldots v_{n}}^{+j_{1} \ldots j_{n}} \partial_{j_{1}}^{\prime v_{1}} \ldots \partial_{j_{n}}^{\prime v_{n}}+\ldots+\ldots\right] \delta\left(\left\{z^{\prime}\right\}\right)
\end{aligned}
$$

If we define

$$
\left[a_{\mu_{1} \ldots \mu_{n}}^{i_{1} \ldots i_{n}}(k), a_{v_{1} \ldots v_{n}}^{+j_{1} \ldots j_{n}}\left(k^{\prime}\right)\right]=f_{\mu_{1} \ldots \mu_{n} v_{1} \ldots v_{n}}^{i_{1} \ldots i_{n} j_{1} \ldots j_{n}}(k) \delta\left(k-k^{\prime}\right)
$$

the commutations relations are

$$
\begin{gathered}
\left.a(k,\{z\}), a^{+}\left(k^{\prime},\left\{z^{\prime}\right\}\right)\right]=\delta\left(k-k^{\prime}\right)\left[f_{0}(k)+f_{\mu_{1} \nu_{1}}^{i_{1} j_{1}}(k) \partial_{i_{1}}^{\mu_{1}} \partial_{j_{1}}^{v_{1}}+\ldots+\ldots\right. \\
\left.f_{\mu_{1} \ldots \mu_{n} \nu_{1} \ldots \nu_{n}}^{i_{1} \ldots i_{n} j_{1} \ldots j_{n}}(k) \partial_{i_{1}}^{\mu_{1}} \ldots \partial_{i_{n}}^{\mu_{n}} \partial_{j_{1}}^{v_{1}} \ldots \partial_{j_{n}}^{v_{n}}+\ldots+\ldots\right] \delta\left(\{z\},\left\{z^{\prime}\right\}\right)
\end{gathered}
$$

\section{$9 \quad$ The Action for the String Field}

\section{The case $\mathbf{n}$ finite}

The action for the free bosonic bradyonic closed string field is:

$$
S_{\text {free }}=\oint_{\left\{\Gamma_{1}\right\}\left\{\Gamma_{2}\right\}} \int_{-\infty}^{\infty} \partial_{\mu} \Phi\left(x,\left\{z_{1}\right\}\right) e^{\left\{z_{1}\right\} \cdot\left\{z_{2}\right\}} \partial^{\mu} \Phi\left(x,\left\{z_{2}\right\}\right) d x d\left\{z_{1}\right\} d\left\{z_{2}\right\}
$$

A possible interaction is given by:

$$
S_{\text {int }}=\lambda \oint_{\left\{\Gamma_{1}\right\}\left\{\Gamma_{2}\right\}\left\{\Gamma_{3}\right\}\left\{\Gamma_{4}\right\}} \oint_{-\infty} \int^{\infty} \Phi\left(x,\left\{z_{1}\right\}\right) e^{\left\{z_{1}\right\} \cdot\left\{z_{2}\right\}} \Phi\left(x,\left\{z_{2}\right\}\right) e^{\left\{z_{2}\right\} \cdot\left\{z_{3}\right\}} \Phi\left(x,\left\{z_{3}\right\}\right) \times
$$




$$
e^{\left\{z_{3}\right\}\left\{z_{4}\right\}} \Phi\left(x,\left\{z_{4}\right\}\right) d x d\left\{z_{1}\right\} d\left\{z_{2}\right\} d\left\{z_{3}\right\} d\left\{z_{4}\right\}
$$

Both, $S_{\text {free }}$ and $S_{\text {int }}$ are non-local as expected.

\section{The case $\mathbf{n} \rightarrow \infty$}

In this case:

$$
\left[S_{\text {free }}=\oint_{\left\{\Gamma_{1}\right\}\left\{\Gamma_{2}\right\}} \oint_{-\infty}^{\infty} \partial_{\mu} \Phi\left(x,\left\{z_{1}\right\}\right) e^{\left\{z_{1}\right\}\left\{z_{2}\right\}} \partial^{\mu} \Phi\left(x,\left\{z_{2}\right\}\right) d x d\left\{\eta_{1}\right\} d\left\{\eta_{2}\right\}\right.
$$

where

$$
\operatorname{d\eta }(z)=\frac{e^{-z^{2}}}{\sqrt{2} \pi}
$$

and

$$
\begin{gathered}
S_{\text {int }}=\lambda \oint_{\left\{\Gamma_{1}\right\}\left\{\Gamma_{2}\right\}\left\{\oint_{3}\right\}\left\{\Gamma_{4}\right\}} \oint_{-\infty} \int^{\infty} \Phi\left(x,\left\{z_{1}\right\}\right) e^{\left\{z_{1}\right\}\left\{\left\{z_{2}\right\}\right.} \Phi\left(x,\left\{z_{2}\right\}\right) e^{\left\{z_{2}\right\}\left\{z_{3}\right\}} \Phi\left(x,\left\{z_{3}\right\}\right) \times \\
e^{\left\{z_{3}\right\} \cdot\left\{z_{4}\right\}} \Phi\left(x,\left\{z_{4}\right\}\right) d x d\left\{\eta_{1}\right\} d\left\{\eta_{2}\right\} d\left\{\eta_{3}\right\} d\left\{\eta_{4}\right\}
\end{gathered}
$$

The convolution of two propagators of the string field is:

$$
\widehat{\Delta}\left(k,\left\{z_{1}\right\},\left\{z_{2}\right\}\right) * \widehat{\Delta}\left(k,\left\{z_{3}\right\},\left\{z_{4}\right\}\right)
$$

where $*$ denotes the convolution of Ultradistributions of Exponential Type on the $k$ variable only. With the use of the result

$$
\frac{1}{\rho} * \frac{1}{\rho}=-\pi^{2} \ln \rho
$$


$\left(\rho=x_{0}^{2}+x_{1}^{2}+x_{2}^{2}+x_{3}^{2}\right.$ in euclidean space $)$

and

$$
\frac{1}{\rho \pm i 0} * \frac{1}{\rho \pm i 0}=\mp i \pi^{2} \ln (\rho \pm i 0)
$$

$\left(\rho=x_{0}^{2}-x_{1}^{2}-x_{2}^{2}-x_{3}^{2}\right.$ in minkowskian space $)$

the convolution of two string field propagators is finite.

\section{Discussion}

We have decided to begin this paper, for the benefit of the reader, with a summary of the main characteristics of Ultradistributions of Exponential Type and their Fourier transform.

We have shown that UET are appropriate for the description in a consistent way string and string field theories. By means of a new Lagrangian for the closed string strictly equivalent to Nambu-Goto Lagrangian we have obtained a movement equation for the field of the string and solve it with the use of CUET We shown that this string field is a linear superposition of CUET. We evaluate the propagator for the string field, and calculate the convolution of two of them, taking into account that string field theory is a non-local theory of UET of an infinite number of complex variables, For 
practical calculations and experimental results we have given expressions that involve only a finite number of variables.

As a final remark we would like to point out that our formulae for convolutions follow from general definitions. They are not regularized expresions 


\section{References}

[1] D. G. Barci, G. Bollini, L. E. Oxman, M. C. Rocca. Int. J. of Theor. Phys. 37, N.12, 3015. (1998).

[2] C. G. Bollini, T. Escobar and M. C. Rocca : Int. J. of Theor. Phys. 38, 2315 (1999).

[3] C. G Bollini and M.C. Rocca : Int. J. of Theor. Phys. 43, 59 (2004).

[4] C. G. Bollini and M. C. Rocca: Int. J. of Theor. Phys. 43, 1019 (2004).

[5] C. G. Bollini and M. C. Rocca:" Convolution of Ultradistributions, Field Theory, Lorentz Invariance and Resonances". Accepted for publication in Int. J. of Theor. Phys. (June 14 (2006)).

[6] J. Sebastiao e Silva : Math. Ann. 136, 38 (1958).

[7] M. Hasumi: Tôhoku Math. J. 13, 94 (1961).

[8] R. F. Hoskins and J. Sousa Pinto: "Distributions, Ultradistributions and other Generalised Functions". Ellis Horwood (1994).

[9] C. G. Bollini, L. E. Oxman, M. C. Rocca. J. of Math. Phys. 35, N. 9, 4429 (1994). 
[10] C. G. Bollini, O. Civitarese, A. L. De Paoli, M. C. Rocca. J. of Math. Phys. 37, 4235 (1996).

[11] A. L. De Paoli, M. Estevez, H. Vucetich, M. C. Rocca.

Infinite Dimensional Analysis, Quantum Probability and Related Topics 4, N.4, 511 (2001).

[12] I. M. Gel'fand and N. Ya. Vilenkin : "Generalized Functions" Vol. 4. Academic Press (1964).

[13] B. Zwiebach:" A First Course in String Theory" Cambridge University Press (2004). 